Published in final edited form as:

Xenobiotica. 2014 January ; 44(1): 28-35. doi:10.3109/00498254.2013.811314.

\title{
Bacterial $\beta$-glucuronidase inhibition protects mice against enteropathy induced by indomethacin, ketoprofen or diclofenac: mode of action and pharmacokinetics
}

\author{
Kyle S. Saitta ${ }^{1}$, Carmen Zhang ${ }^{1}$, Kang Kwang Lee ${ }^{1}$, Kazunori Fujimoto ${ }^{1}$, Matthew R. \\ Redinbo $^{2}$, and Urs A. Boelsterli ${ }^{1}$ \\ ${ }^{1}$ Department of Pharmaceutical Sciences, University of Connecticut School of Pharmacy, Storrs, \\ Connecticut, USA \\ ${ }^{2}$ Department of Chemistry, University of North Carolina, Chapel Hill, North Carolina, USA
}

\section{Abstract}

1. We have previously demonstrated that a small molecule inhibitor of bacterial $\beta$ glucuronidase (Inh-1; [1-((6,8-dimethyl-2-oxo-1,2-dihydroquinolin-3-yl)-3-(4ethoxyphenyl)-1-(2-hydroxyethyl)thiourea]) protected mice against diclofenac (DCF)induced enteropathy. Here we report that Inh-1 was equally protective against small intestinal injury induced by other carboxylic acid-containing non-steroidal antiinflammatory drugs (NSAIDs), indomethacin (10 mg/kg, ip) and ketoprofen $(100 \mathrm{mg} / \mathrm{kg}$, ip).

2. Inh-1 provided complete protection if given prior to DCF ( $60 \mathrm{mg} / \mathrm{kg}$, ip), and partial protection if administered 3-h post-DCF, suggesting that the temporal window of mucosal protection can be extended for drugs undergoing extensive enterohepatic circulation.

3. Pharmacokinetic analysis of Inh-1 revealed an absolute bioavailability $(F)$ of $21 \%$ and a short $t_{1 / 2}$ of $<1 \mathrm{~h}$. This low $F$ was shown to be due to hepatic first-pass metabolism, as confirmed with the pan-CYP inhibitor, 1-aminobenzotriazole.

4. Using the fluorescent probe 5 (and 6)-carboxy-2', $7^{\prime}$-dichlorofluorescein, we demonstrated that Inh-1 did not interfere with hepatobiliary export of glucuronides in gall bladdercannulated mice.

5. These data are compatible with the hypothesis that pharmacological inhibition of bacterial $\beta$-glucuronidase-mediated cleavage of NSAID glucuronides in the small intestinal lumen can protect against NSAID-induced enteropathy caused by locally high concentrations of NSAID aglycones.

\section{Keywords}

Acyl glucuronide; gastrointestinal toxicity; glucuronide conjugate; Inhibitor-1; NSAIDs; phenol glucuronide; small intestinal ulcers

\footnotetext{
(C) 2014 Informa UK Ltd.

Address for correspondence: Urs A. Boelsterli, PhD, Professor and Boehringer Ingelheim Endowed Chair in Mechanistic Toxicology, Department of Pharmaceutical Sciences, University of Connecticut School of Pharmacy, 69 North Eagleville Road, Unit 3092, Storrs, CT 06269-3092, USA. Tel: 860-486-8087. Fax: 860-486-5792. urs.boelsterli@uconn.edu.

Declaration of interest

The authors report no declarations of interest.
} 


\section{Introduction}

Non-steroidal anti-inflammatory drugs (NSAIDs), despite being generally safe drugs, have the potential to produce widespread gastrointestinal adverse drug reactions. These include not only gastroduodenal injury but also mucosal erosions and ulceration in the small intestine (Scarpignato \& Hunt, 2010; Wallace, 2012). Although often clinically silent initially, when taken long-term, the complications associated with NSAID-induced enteropathy (bleeding, protein loss, strictures, and, rarely, perforations) may become serious (Bjarnason, 2013). To date, there are no clinically approved effective therapies available to prevent or treat NSAID-induced enteropathy.

One reason for the lack of effective therapies is our incomplete understanding of the mechanisms underlying NSAID enteropathy. It has become clear that the pathogenesis is a multi-step sequence of events involving multiple mechanisms (Boelsterli et al., 2013). In the mucosal epithelia (enterocytes), NSAIDs or their reactive metabolites can cause endoplasmic reticulum stress and mitochondrial stress, which can lead to epithelial cell injury and cell death. This is followed by an increase in the epithelial permeability, bacterial migration into deeper layers of the mucosa and activation of an inflammatory cascade mediated by toll-like receptors, and amplification of the injury by an inflammatory response, resulting in deep ulceration of the mucosa. These events can be modulated by other factors (including prostaglandin synthase inhibition by the NSAIDs).

However, at the top of these cascades of events, a crucial determinant is the hepatobiliary export of NSAID glucuronides, which is common for many NSAIDs. Specifically, carboxylic acid-containing NSAIDs can form acyl glucuronides, and ring hydroxylation can lead to phenol glucuronides, or a combination of the two glucuronidation steps. Both acyl glucuronides and phenol glucuronides of diclofenac (DCF) have recently been identified in mice following a single administration of the drug (Sarda et al., 2012). These glucuronides are delivered to the small intestinal lumen, where they are cleaved by bacterial $\beta$ glucuronidase, releasing the aglycones. The enterocytes thus become exposed to relatively high concentrations of the free parent and oxidative metabolites, where they are believed to cause mitochondrial and ER stress.

We have previously demonstrated in a mouse model of DCF-induced enteropathy (RamirezAlcantara et al., 2009) that inhibition of $\beta$-glucuronidase with Inhibitor-1 (Inh-1), a small molecule inhibitor of the bacteria-specific forms of $\beta$-glucuronidase (Wallace et al., 2010), can greatly reduce the extent of DCF-induced enteropathy (LoGuidice et al., 2012). However, it is not known whether this protective effect is selective for DCF or more generally applicable to other cholephilic NSAIDs. Furthermore, the mode of action and the disposition of these $\beta$-glucuronidase inhibitors are incompletely characterized. Therefore, this follow-up study was aimed at exploring whether the same mechanism of protection could be expanded to other carboxylic acid-containing NSAIDs and at further characterizing the mode of action of Inh-1, including its pharmacokinetic properties and temporal window of action.

\section{Materials and methods}

\section{Chemicals and reagents}

Indomethacin, ketoprofen, diclofenac, 5 (and 6)-carboxy-2 $2^{\prime}, 7^{\prime}$-dichlorofluorescein (CDF), 5 (and 6)-carboxy-2 $2^{\prime}, 7^{\prime}$-dichlorofluorescein diacetate (CDFDA), and cyclosporin A were obtained from Sigma-Aldrich (St. Louis, MO). Inhibitor-1 [1-((6,8-dimethyl-2-oxo-1,2dihydroquinolin-3-yl)methyl)-3-(4-ethoxyphenyl)-1-(2-hydroxyethyl)thiourea] was obtained 
from Asinex, Inc. (Winston-Salem, NC); its structure was confirmed by MS, and the purity was $99 \%$. Solutol HS-15 was from BASF (Ludwigshafen, Germany).

\section{Animals and drug treatment}

Male C57BL/6J mice were obtained from The Jackson Laboratory (Bar Harbor, ME). The mice were acclimatized for 1 week and were 10 to 12 weeks of age at the start of the experiments. The animals were kept on a 14/10-h light/dark cycle. They received mouse chow (Teklad Global Rodent Diet; Harlan Laboratories, Boston, MA) and water ad libitum. All studies were approved by the Institutional Animal Care and Use Committee of the University of Connecticut. Indomethacin, ketoprofen and diclofenac were dissolved in 10\% (in phosphate-buffered saline) Solutol HS-15 solution and administered intraperitoneally in a volume of $10 \mu \mathrm{l} / \mathrm{g}$ body weight. For pretreatment studies, Inh- 1 or vehicle $(0.5 \%$ aqueous methyl cellulose) was administered by oral gavage b.i.d. (10 $\mu \mathrm{g}$ per mouse), starting 1 day before indomethacin or ketoprofen administration and with the last dose given $1 \mathrm{~h}$ before indomethacin or ketoprofen; for post-treatment studies, Inh-1 or vehicle was administered as a single dose by oral gavage (10 $\mu \mathrm{g}$ per mouse) $3 \mathrm{~h}$ after DCF. The dosage regimen for Inh- 1 was adopted from a previously published study (Wallace et al., 2010). For the toxicopathology studies, 5-11 mice per treatment group were used, and for the kinetic study, nine mice per treatment group (three mice for identical time points). The doses for the three NSAIDs (DCF, $60 \mathrm{mg} / \mathrm{kg}$; indomethacin, $10 \mathrm{mg} / \mathrm{kg}$; ketoprofen, $100 \mathrm{mg} / \mathrm{kg}$ ) were selected from previously published studies (Fukumoto et al., 2011; Ramirez-Alcantara et al., 2009); after correction with the interspecies dose scaling factor ( $\times 12$ for mouse) (Kirman et al., 2005), they are comparable to the human therapeutic doses. The intraperitoneal route was selected to ensure maximal absorption and to minimize drug-drug interactions during administration; previous data have shown that DCF administered either intraperitoneally or orally produced a similar extent of enteropathy in rats, due to the critical role of hepatobiliary excretion of glucuronoconjugates (Seitz \& Boelsterli, 1998).

\section{Assessment of small intestinal injury}

Mice were euthanized by $\mathrm{CO}_{2}$ inhalation at $18 \mathrm{~h}$ after indomethacin, ketoprofen or DCF. A midline incision was made, and blood was obtained via cardiac puncture. Serum was prepared and frozen at $-80{ }^{\circ} \mathrm{C}$ until use for analysis. The entire small intestine (from the gastroduodenal junction to the ileocecal junction) was removed and opened longitudinally along the antimesenteric side. The tissue was rinsed in ice-cold phosphate-buffered saline and incubated for $15 \mathrm{~min}$ in $1 \mathrm{mM}$ nitroblue tetrazolium (NBT) solution containing $16 \mathrm{mM}$ HEPES-NaOH, $125 \mathrm{mM} \mathrm{NaCl}$ buffer, $3.5 \mathrm{mM} \mathrm{KCl}$ and $10 \mathrm{mM}$ glucose. Next, the tissues were fixed in $10 \%$ zinc formalin for $24 \mathrm{~h}$, washed and transferred to $70 \%$ ethanol. The intestine was metrically divided into four quartiles of equal length and evaluated at $10 \times$ magnification for quantitative and qualitative analysis of mucosal injury, and the lesions were assigned to the respective quartiles. In addition, the total area of the lesions was quantified by ImageJ software (NIH). Images were taken using an Olympus SZX16 microscope with an Olympus DP72 camera.

\section{Hepatocyte culture and exposure to chemicals}

Hepatocytes were isolated from 25 - to $30-\mathrm{g}$ male C57BL/6J mice by collagenase perfusion through the inferior vena cava, using Percoll purification (Fujimoto et al., 2009). The cells were resuspended in complete Williams' medium E containing 10\% fetal calf serum, $100 \mathrm{U} /$ $\mathrm{mL}$ penicillin and $100 \mu \mathrm{g} / \mathrm{mL}$ streptomycin. Cell viability was greater than $95 \%$ as determined by trypan blue exclusion. Hepatocytes were plated in 48 -well plates $(8.0 \times$ $10^{4}$ cells per well). Plates were coated with $50 \mu \mathrm{g} / \mathrm{mL}$ rat-tail collagen. Hepatocytes were allowed to attach for $3 \mathrm{~h}$ in a humidified atmosphere of $5 \% \mathrm{CO}_{2}, 95 \%$ air at $37{ }^{\circ} \mathrm{C}$. 
Subsequently, hepatocytes were washed once and then incubated in the same medium. For all incubations with chemicals, serum- and antibiotic-free culture medium was used. Release of lactate dehydrogenase (LDH) into the extracellular medium was taken as an indicator of cytotoxicity. LDH activity was determined with the CytoTox-One Homogeneous Membrane Integrity assay (Promega, Madison, WI) and expressed as percentage of activity present in the medium as compared to the total intra- and extracellular LDH.

\section{Pharmacokinetic profiling of Inhibitor-1}

Mice were treated with a single dose (3 mg/kg) of Inh-1 both orally (in $0.5 \%$ sodium carboxymethyl cellulose and $0.1 \% \mathrm{v} / \mathrm{v}$ Tween 80 in water; administered in a volume of 10 $\mathrm{ml} / \mathrm{kg}$ ) or intravenously (in 7.5\% $N$-methyl pyrrolidone and 40\% PEG-400 in water; administered in a volume of $5 \mathrm{ml} / \mathrm{kg}$ ). 1-Aminobenzotriazole (ABT) was administered by oral gavage $(50 \mathrm{mg} / \mathrm{kg}$ in water; $10 \mathrm{ml} / \mathrm{kg}$ ). Blood samples (approx. $60 \mu \mathrm{l}$ ) were collected from a set of three mice at pre-dose, $0.25,0.5,1,2,4,6,8$ and $24 \mathrm{~h}$ (oral) and pre-dose, $0.08,0.25,0.5,1,2,4,8$ and $24 \mathrm{~h}$ post-dose (intravenous). Immediately after collection of blood, plasma was prepared and stored at $-70{ }^{\circ} \mathrm{C}$ until analysis. All samples were processed for analysis by precipitation using acetonitrile and analyzed by LC-MS-MS (Sai Advantium Pharma Ltd., Pune, India). In brief, a Merck Chromolith Flash column $(4.6 \times 25 \mathrm{~mm} ; 5 \mu \mathrm{m}$ particle size) was used. The mobile phase consisted of acetonitrile (A) and $0.1 \%$ formic acid (B, gradient mode), with a flow rate of $800-1000 \mu \mathrm{l} / \mathrm{min}$ at $40^{\circ} \mathrm{C}$. Mass spectrometry was performed with an API 4000 (AB Sciex, Framingham, MA) equipped with a turbo ion spray source. Validation of the analytical method revealed that the limit of detection was $1.01 \mathrm{ng} /$ $\mathrm{mL}$, the standard curve accuracy was 84.3-116.4 of the nominal concentration, the slope and $R$ of the standard curve were 0.00135 and 0.9925 , respectively; imipramine was used as internal standard. The Non-compartmental-Analysis module in WinNonlin (version 5.2; Pharsight, Mountain View, CA) was used to assess the pharmacokinetic parameters.

\section{Determination of hepatobiliary CDF excretion}

Mice were administered Inh- 1 or vehicle ( $0.5 \%$ methyl cellulose) by oral gavage b.i.d. (10 $\mu \mathrm{g}$ per mouse) for two days with the last dose given $1 \mathrm{~h}$ before anesthesia. Cyclosporin A was dissolved in 10\% (in phosphate-buffered saline) Solutol HS-15 solution and administered intraperitoneally $(50 \mathrm{mg} / \mathrm{kg}) 1 \mathrm{~h}$ before anesthesia as a positive control. Mice were anesthetized using ketamine $(100 \mathrm{mg} / \mathrm{kg}$, ip) and xylazine $(10 \mathrm{mg} / \mathrm{kg}$, ip). An incision was made along the linea alba to expose the gallbladder. The animals were kept at constant body temperature of $37^{\circ} \mathrm{C}$ with the use of a heat lamp. A 4-0 nylon ligature was tied distal to the gallbladder, which was then punctured with PE10 tubing. The nylon ligature was used to hold the cannula in place. Mice were then administered $1.5 \mu \mathrm{mol} /$ mouse of CDFDA in $10 \%$ (in saline) DMSO intraperitoneally. Bile was collected in $0.6 \mathrm{ml}$ Eppendorf tubes at 10min intervals for $60 \mathrm{~min}$. CDF concentrations in bile (diluted with saline) were determined by spectrofluorometry $\left(\lambda_{\mathrm{ex}} / \lambda_{\mathrm{em}}, 505 / 523 \mathrm{~nm}\right)$ in a Tecan multiwell plate reader (Maennedorf, Switzerland). Standard curves of CDF were prepared daily and were linear with concentrations.

\section{Statistical analysis}

All data were expressed as mean \pm SD. If there was normal distribution, a standard analysis of variance used, followed by Dunnett's test for multiple comparisons versus the control group. When normality failed, a Kruskal-Wallis one-way analysis of variance on ranks was used followed by Dunn's test for multiple comparison versus the control group. A $p$ value of $<0.05$ was considered significant. 


\section{Results}

\section{Jejunal injury induced by indomethacin or ketoprofen is attenuated by Inhibitor-1 pretreatment}

We have previously demonstrated that the bacterial $\beta$-glucuronidase inhibitor Inh- 1 affords protection against enteropathy induced by DCF (LoGuidice et al., 2012). To ascertain whether this is a general mechanism for carboxylic acid-containing NSAIDs, as opposed to an unrelated DCF-specific effect, we assessed the enteric-protective effects of Inh-1 against indomethacin- or ketoprofen-induced small intestinal injury. Both indomethacin and ketoprofen are carboxylic acid-containing NSAIDs that are conjugated to glucuronic acid in the liver, followed by hepatobiliary excretion of the glucuronides and enterohepatic circulation of the aglycones (Dial et al., 2008; Duggan et al., 1975; Evans, 1992; Julou et al., 1976). We found that mice which received a single dose of indomethacin alone $(10 \mathrm{mg} / \mathrm{kg}$, ip) developed marked mucosal ulceration at the mesenteric side of the small intestine, with a typical distribution pattern exhibiting the majority of lesions in quartiles 2 and 3 (Figure $1 \mathrm{~A}-\mathrm{C}$ ), whereas vehicle-treated mice were completely devoid of any ulceration (not shown). In contrast, pretreatment of mice with Inh-1 (10 $\mu \mathrm{g} /$ mouse, po, bid, for 2 days) greatly decreased the mean number and total area of indomethacin-induced intestinal injury by $90 \%$ and $89 \%$, respectively. In addition, following incubation of the jejunal mucosa with nitroblue tetrazolium (NBT), large areas of the luminal surface of the indomethacin-treated mice developed a blue color (indicative of NBT reduction to a blue non-soluble formazan), while the mucosa of the Inh-1/indomethacin co-treated mice remained unstained. This suggests that the oxidoreductive stress induced by indomethacin was largely prevented by Inh-1 pretreatment.

Similarly, administration of ketoprofen alone $(100 \mathrm{mg} / \mathrm{kg}$, ip) caused mild jejunal ulceration in some mice; however, 5/10 mice were non-responders (Figure 1D). Again, Inh-1 significantly attenuated the jejunal lesions induced by ketoprofen; there was only one small ulcer detected in 1/11 co-treated mice. Taken together, these data indicate that the protection afforded by the $\beta$-glucuronidase inhibitor, Inh-1, is not specific for DCF but that it can be expanded to other, structurally distinct NSAIDs that are lipophilic, weakly acidic carboxylic acid-containing molecules subject to hepatic glucuronoconjugation, hepatobiliary excretion and enterohepatic circulation.

\section{The time window for Inh-1 co-administration with NSAIDs is critical}

The putative mode of Inh-1-mediated protection against NSAID enteropathy is inhibition of bacterial $\beta$-glucuronidase in the GI lumen, which implies that the inhibitor be administered to mice prior to the delivery of glucuronides via bile. Therefore, we next aimed at determining whether post-treatment with the inhibitor, as opposed to pre-treatment, would still be effective in alleviating the mucosal injury. The rationale for this was based on the pharmacokinetic properties of acidic NSAIDs, which undergo enterohepatic cycling, thus delaying the clearance of the drugs. Mice were treated with a single ulcerogenic dose of DCF $(60 \mathrm{mg} / \mathrm{kg}$, ip), followed by a single dose of Inh-1 or vehicle $3 \mathrm{~h}$ post-DCF administration. The 3-hour time point was chosen because the first cycle of enterohepatic circulation is completed at this time and the highest rates of biliary output of diclofenac conjugates have occurred in this time interval (Reuter \& Wallace, 1999; Seitz et al., 1998), while at the same time macroscopically defined ulcers have not yet developed (RamirezAlcantara et al., 2009). We found that post-treatment with Inh-1 reduced the average ulcer number per mouse by $71 \%$ as compared to DCF controls, while the average area of total ulcers per mouse was not significantly different from that in mice receiving DCF alone (Figure 2). These data indicate that, although the degree of protection is lower than that in Inh-1-pretreated mice (LoGuidice et al., 2012), post-treatment with the bacterial $\beta$ - 
glucuronidase inhibitor still provides partial protection, likely due to the extensive enterohepatic circulation that is still ongoing at $3 \mathrm{~h}$ after a dose of DCF.

\section{Inh-1 does not attenuate acute lethal cell injury in vitro}

To address the possibility that Inh-1 may be cytoprotective by inhibiting a pathway downstream of $\beta$-glucuronidase, including a direct inhibitory effect on NSAID-induced cell death, we exposed cultured primary mouse hepatocytes to DCF in the presence or absence of Inh-1. As shown in Figure 3, a high cytotoxic concentration of DCF $(1000 \mu \mathrm{M})$ induced significant increases in LDH release from the cells, which is consistent with previous reports (Kretz-Rommel \& Boelsterli, 1993; Lim et al., 2006). Inh-1 (100 $\mu$ M, a non-toxic concentration that did not significantly increase LDH release as compared to solvent controls) did not alter the concentration-toxic response curve, indicating that the $\beta$ glucuronidase inhibitor is unlikely to interfere with the cell death-inducing pathways involved in DCF cytotoxicity. In aqueous solutions containing $>100 \mu \mathrm{M}$ of Inh-1 (from stock solutions in DMSO), Inh-1 precipitates as solid material, thus limiting cell-based studies with higher concentrations.

\section{Pharmacokinetic analysis of Inhibitor-1 in mice}

To better characterize the inhibitory potential of the orally administered bacterial $\beta$ glucuronidase inhibitor, we performed a kinetic study to obtain the plasma concentration over time profile of Inh-1. First, plasma concentrations of Inh-1 were serially determined following a single oral administration (Figure 4). Maximal plasma levels were attained after $30 \mathrm{~min}$, and the compound was rapidly cleared (Table 1). To determine the absolute bioavailability $(F)$ of Inh-1, the AUC was compared with that of a single intravenous administration of Inh-1. We found that $F$ was $21 \%$ (Table 1). To ascertain whether this poor oral bioavailability was caused by poor absorption or by first-pass metabolism, or both, we pretreated another set of mice with 1-aminobenzotriazole (ABT, $50 \mathrm{mg} / \mathrm{kg}$ ) (Caldwell et al., 2005). This non-toxic dose of ABT is sufficient to inhibit a number of CYPs in mice (Balani et al., 2004). We found that, after oral administration of Inh-1, ABT increased $F$ to $99 \%$, indicating that Inh-1 is almost fully absorbed in the GI. The data therefore suggest that the poor absolute bioavailability of Inh-1 was due to CYP-mediated metabolic clearance. Specifically, we found that ABT also inhibited Inh- 1 clearance when the $\beta$-glucuronidase inhibitor was given intravenously rather than orally, strongly suggesting that the CYPmediated metabolism of Inh-1 mostly occurred in the liver, not in the gastrointestinal tract.

\section{Effect of Inhibitor-1 on hepatobiliary conjugate excretion}

Because Inh-1 was found to be well absorbed and to be metabolized in the liver, we next conducted a series of experiments to ascertain whether the compound might interfere with the hepatobiliary excretion of cholephilic compounds, including NSAID glucuronides, and whether this would be a factor that contributes to the protective effects of the $\beta$ glucuronidase inhibitors. Because glucuronides are primarily exported into bile by the conjugate export pump, Mrp2 (Abcc2), we used 5 (and 6)-carboxy-2' $7^{\prime}$-dichlorofluorescein (CDF), a non-metabolizable fluorescent probe that has found wide use as a model substrate for Mrp2 transport function in vitro and in vivo (Colombo et al., 2012; Zamek-Gliszczynski et al., 2003). Because hepatic extraction of free CDF from the blood is low in mice, we administered $\mathrm{CDF}$ as its diacetate ester to increase hepatic delivery and to circumvent hepatic uptake as a rate-limiting factor (Nezasa et al., 2006). CDFDA is avidly taken up by the liver by diffusion, and rapidly hydrolyzed in the liver. We found that, in the anesthetized and gall bladder-cannulated mice, CDF-related fluorescence appeared in bile after a lag time of $10 \mathrm{~min}$, reaching a peak at $30 \mathrm{~min}$ and starting to decline afterwards (Figure 5).

Pretreatment with Inh-1 had no significant effect on the excretion rates of CDF. In contrast, 
cyclosporin A (CsA), which was used as a positive control, caused significant reductions in CDF biliary concentrations. Inh-1 also did not have any significant effect on the total amount of bile secreted; in vehicle controls, bile flow was stable and ranged from 14 to 18 $\mu 1 / 10$ min interval. In contrast, as expected, CsA reduced bile flow by $>50 \%$ (data not shown). Collectively, these data suggest that Inh-1 is unlikely to negatively affect canalicular glucuronide export function in mouse liver.

\section{Discussion}

The gut microbiota plays an eminent role in the metabolism and bioactivation of xenobiotics (Wilson \& Nicholson, 2009). The role of one toxicologically relevant intestinal bacterial enzyme, $\beta$-glucuronidase (encoded by the gus gene), has recently been revisited as newly developed specific bacterial $\beta$-glucuronidase inhibitors became available (Wallace et al., 2010). The data presented in this study are consistent with our hypothesis that oral administration of one of these bacteria-specific inhibitors of $\beta$-glucuronidase (Inh-1) can greatly attenuate inflammatory ulceration of the small intestine by preventing enzymatic cleavage of the glucuronides and release of the aglycones in the jejunal lumen. The results confirm and extend those reported in a previous study (LoGuidice et al., 2012) and include other acidic and potentially ulcerogenic NSAIDs (indomethacin and ketoprofen). The conclusion that Inh-1 exerts its effect in the gut lumen and not at a more proximal or more distal site is supported by the findings that Inh-1 does not interfere with the hepatobiliary excretion of glucuronides nor directly inhibit the toxic response of target cells exposed to the aglycone. Furthermore, all three NSAIDs investigated in this study share a number of common structural and pharmacokinetic features, including a carboxylic acid moiety and the propensity to undergo glucuronide conjugation, hepatobiliary excretion of the glucuronides, and enterohepatic circulation of the aglycones. Thus, the major pharmacokinetic determinant of the GI toxicity of these compounds is the $\beta$-glucuronidase-mediated release of the aglycone(s) that are subsequently taken up by the enterocytes where they initiate a cascade of events leading to cell injury (Boelsterli et al., 2013).

The quantitative and qualitative profile of NSAID glucuronides other than acyl glucuronides in bile and the intestinal lumen are currently not known. However, a recent linear ion trap mass spectrometric analysis has revealed that DCF acyl glucuronide formation may be a minor route of DCF glucuronidation in mice and that glucuronides formed from the ringhydroxylated metabolites may be quantitatively more important (Sarda et al., 2012). Interestingly, the study also demonstrated that glucuronidation of the 4'-hydroxy and 5hydroxy metabolites was directed at the formation of ether rather than acyl conjugates. Phenolic (ether) glucuronides, similar to the acyl glucuronides, are also excellent substrates for bacterial $\beta$-glucuronidase (Wallace et al., 2010). Whether and how these oxidative metabolites of DCF are taken up by enterocytes is not clear; however, it has been demonstrated that intestinal CYP-mediated metabolism is critical for enteropathy to develop, because intestine-specific genetic elimination of CYP reductase resulted not only in a great decrease of intestinal DCF oxidative metabolite formation, but also in full protection against DCF-induced enteropathy (Zhu \& Zhang, 2012).

Pharmacologic inhibition of NSAID-induced enteropathy can be aimed at different molecular targets. For example, indomethacin enteropathy has recently been reported to be attenuated by co-administration of hemin, a heme oxygenase- 1 inducer (Yoriki et al., 2012). Hemin clearly interferes with the second hit during the pathogenesis of NSAID-induced small intestinal injury, i.e. the mucosal inflammatory response. Here, we demonstrate that inhibition of the release of the parent indomethacin (or an oxidative metabolite) from the glucuronide conjugate, which causes the first hit, is also a highly effective mechanism to protect the jejunal mucosa. However, for indomethacin, the biliary metabolite profile is less 
well known as compared to that of DCF. Apart from CYPs, other enzymes could play a key role in the bio-activation of the compound. For example, a major indomethacin metabolite, desmethyldeschlorobenzoyl indomethacin, has been shown to be activated in vitro by neutrophils via myeloperoxidase-catalyzed oxidation to a reactive intermediate, possibly an iminoquinone (Ju \& Uetrecht, 1998). To what extent this route would play a role in indomethacin enteropathy is currently not known.

With a view to potential clinical applications of such small molecule inhibitors of bacteriaspecific $\beta$-glucuronidase against NSAID-associated small intestinal injury, a number of points have to be considered. First, because the mode of action of the inhibitor is based on an early event (prior to intestinal absorption of the NSAIDs), such a mechanism would imply that the $\beta$-glucuronidase inhibitor would find a potential role as a prophylactic, rather than a therapeutic, agent. Although our data demonstrate that, if given $3 \mathrm{~h}$ post-DCF, the protective effects of Inh-1 is still partially retained, the inhibitor needs to be administered prior to the induction of jejunal ulceration. Second, ideally such an inhibitor should be either minimally absorbed and stay in the intestinal lumen, where it can react with the luminal bacterial enzyme, or it should be rapidly eliminated in order not to interfere with endogenous glucuronide homeostasis. For Inh-1, we show that even in view of its short systemic half-life $(<1 \mathrm{~h})$ and rapid hepatic clearance, the inhibitor prevents the progression of the disease in the mouse model. Finally, it must be assessed to what extent the mouse model reflects the human situation with respect to the extent of enterohepatic circulation; unfortunately quantitative comparable data are currently not available.

\section{Conclusions}

Inhibitor-1 (Inh-1), a selective small-molecule inhibitor of bacteria-specific $\beta$-glucuronidase, protects against small intestinal injury induced by the carboxylic acid-containing NSAIDs diclofenac, indomethacin or ketoprofen by preventing the hydrolytic cleavage of the acyl glucuronides and/or phenol glucuronides that are excreted into bile. These results are presently limited to the mouse model, and the translation of these observations to humans remains uncertain. However, bacterial $\beta$-glucuronidase could be explored as a new pharmacologic target in the development of GI-protective drugs against NSAID-induced enteropathy.

\section{Acknowledgments}

We thank Dr R. Kanadia, University of Connecticut, for technical help with the morphometric tissue analysis.

This work was supported by National Institutes of Health [grant CA98468] and the University Cancer Research Fund of North Carolina (to M.R.R.); the Boehringer Ingelheim Endowed Chair in Mechanistic Toxicology; and a research grant from Helsinn Healthcare SA, Switzerland (to U.A.B.).

\section{References}

Balani SK, Li P, Nguyen J, et al. Effective dosage regimen of 1-aminobenzotriazole for inhibition of antipyrene clearance in guinea pigs and mice using serial sampling. Drug Metab Disp. 2004; 32:1092-5.

Bjarnason I. Gastrointestinal safety of NSAIDs and over-the-counter analgesics. Int J Clin Pract. 2013; 67:37-42.

Boelsterli UA, Redinbo MR, Saitta K. Multiple NSAID-induced hits injure the small intestine: underlying mechanisms and novel strategies. Toxicol Sci. 2013; 131:654-67. [PubMed: 23091168]

Caldwell GW, Ritchie DM, Masucci JA, et al. The use of the suicide CYP450 inhibitor ABT for distinguishing absorption and metabolism processes in in-vivo pharmacokinetic screens. Eur J Drug Metab Pharmacokinet. 2005; 30:75-83. [PubMed: 16010865] 
Colombo F, Armstrong C, Duan J, Rioux N. A high throughput in vitro mrp2 assay to predict in vivo biliary excretion. Xenobiotica. 2012; 42:157-63. [PubMed: 21961530]

Dial EJ, Darling RL, Lichtenberger LM. Importance of biliary excretion of indomethacin in gastrointestinal and hepatic injury. J Gastroenterol Hepatol. 2008; 23:e384-9. [PubMed: 18086111]

Duggan DE, Hooke KF, Noll RM, Kwan KC. Enterohepatic circulation of indomethacin and its role in intestinal irritation. Biochem Pharmacol. 1975; 25:1749-54. [PubMed: 823946]

Evans AM. Enantioselective pharmacodynamics and pharmacokinetics of chiral non-steroidal antiinflammatory drugs. Eur J Clin Pharmacol. 1992; 42:237-56. [PubMed: 1577041]

Fujimoto K, Kumagai K, Ito K, et al. Sensitivity of liver injury in heterozygous Sod 2 knockout mice treated with troglitazone or acetaminophen. Toxicol Pathol. 2009; 37:193-200. [PubMed: 19332662]

Fukumoto K, Naito Y, Takagi T, et al. Role of tumor necrosis factor-a in the pathogenesis of indomethacin-induced small intestinal injury in mice. Int J Molec Med. 2011; 27:353-9. [PubMed: 21249312]

Ju C, Uetrecht J. Oxidation of a metabolite of indomethacin (desmethyldeschlorobenzoylindomethacin) to reactive intermediates by activated neutrophils, hypochlorous acid, and the myeloperoxidase system. Drug Metab Disp. 1998; 26:676-80.

Julou L, Guyonnet JC, Ducrot R, et al. Ketoprofen (19.583 R.P.) (2-(3-benzoylphenyl)-propionic acid). Main pharmacological properties - outline of toxicological and pharmacokinetic data. Scand $\mathrm{J}$ Rheumatol suppl. 1976; 14:33-44. [PubMed: 824723]

Kirman CR, Sweeney LM, Meek ME, Gargas ML. Assessing the dose-dependency of allometric scaling performance using physiologically based pharmacokinetic modeling. Reg Toxicol Pharmacol. 2005; 38:345-67.

Kretz-Rommel A, Boelsterli U. Diclofenac covalent protein binding is dependent on acyl glucuronide formation and is inversely related to acute cell injury in cultured rat hepatocytes. Toxicol Appl Pharmacol. 1993; 120:155-61. [PubMed: 8511777]

Lim MS, Lim PLK, Gupta R, Boelsterli UA. Critical role of free cytosolic calcium, but not uncoupling, in mitochondrial permeability transition and cell death induced by diclofenac oxidative metabolites in immortalized human hepatocytes. Toxicol Appl Pharmacol. 2006; 217:322-31. [PubMed: 17097122]

LoGuidice A, Wallace BD, Bendel L, et al. Pharmacologic targeting of bacterial $\beta$-glucuronidase alleviates nonsteroidal anti-inflammatory drug-induced enteropathy in mice. J Pharmacol Exp Ther. 2012; 341:447-54. [PubMed: 22328575]

Nezasa K, Tian X, Zamek-Gliszczynski MJ, et al. Altered hepatobiliary disposition of 5 (and 6)carboxy-2',7'-dichlorofluorescein in Abcg2 (Bcrp1) and Abcc2 (Mrp2) knockout mice. Drug Metab Disp. 2006; 34:718-23.

Ramirez-Alcantara V, LoGuidice A, Boelsterli UA. Protection from diclofenac-induced small intestinal injury by the JNK inhibitor SP600125 in a mouse model of NSAID-associated enteropathy. Am J Physiol (Gastrointest Liver Physiol). 2009; 297:G990-8. [PubMed: 20501447]

Reuter BK, Wallace JL. Phosphodiesterase inhibitors prevent NSAID enteropathy independently of effects on TNF-a release. Am J Physiol (Gastrointest Liver Physiol). 1999; 40:G847-54.

Sarda S, Page C, Pickup K, et al. Diclofenac metabolism in the mouse: novel in vivo metabolites identified by high performance liquid chromatography coupled to linear ion trap mass spectrometry. Xenobiotica. 2012; 42:179-94. [PubMed: 21955289]

Scarpignato C, Hunt RH. Nonsteroidal antiinflammatory drug-related injury to the gastrointestinal tract: clinical picture, pathogenesis, and prevention. Gastroenterol Clin N Am. 2010; 39:433-64.

Seitz S, Boelsterli UA. Diclofenac acyl glucuronide, a major biliary metabolite, is directly involved in small intestinal injury in rats. Gastroenterology. 1998; 115:1476-82. [PubMed: 9834275]

Seitz S, Kretz-Rommel A, Oude Elferink RPJ, Boelsterli UA. Selective protein adduct formation of diclofenac glucuronide is critically dependent on the rat canalicular conjugate export pump (Mrp2). Chem Res Toxicol. 1998; 11:513-19. [PubMed: 9585482]

Wallace BD, Wang H, Lane KT, et al. Alleviating cancer drug toxicity by inhibiting a bacterial enzyme. Science. 2010; 330:831-5. [PubMed: 21051639] 
Wallace JL. NSAID gastropathy and enteropathy: distinct pathogenesis likely necessitates distinct prevention strategies. Br J Clin Pharmacol. 2012; 165:67-74.

Wilson ID, Nicholson JK. The role of gut microbiota in drug response. Curr Pharmaceut Design. 2009; 15:1519-23.

Yoriki H, Naito Y, Takagi T, et al. Hemin ameliorates indomethacin-induced small intestinal injury in mice through the induction of heme oxygenase-1. J Gastroenterol Hepatol. 2012; 28:632-8. [PubMed: 23216607]

Zamek-Gliszczynski MJ, Xiong H, Patel NJ, et al. Pharmacokinetics of 5 (and 6)-carboxy-2',7'dichlorofluorescein and its diacetate promoiety in the liver. J Pharmacol Exp Ther. 2003; 304:8019. [PubMed: 12538836]

Zhu Y, Zhang QY. Role of intestinal cytochrome P450 enzymes in diclofenac-induced toxicity in the small intestine. J Pharmacol Exp Ther. 2012; 343:362-70. [PubMed: 22892338] 

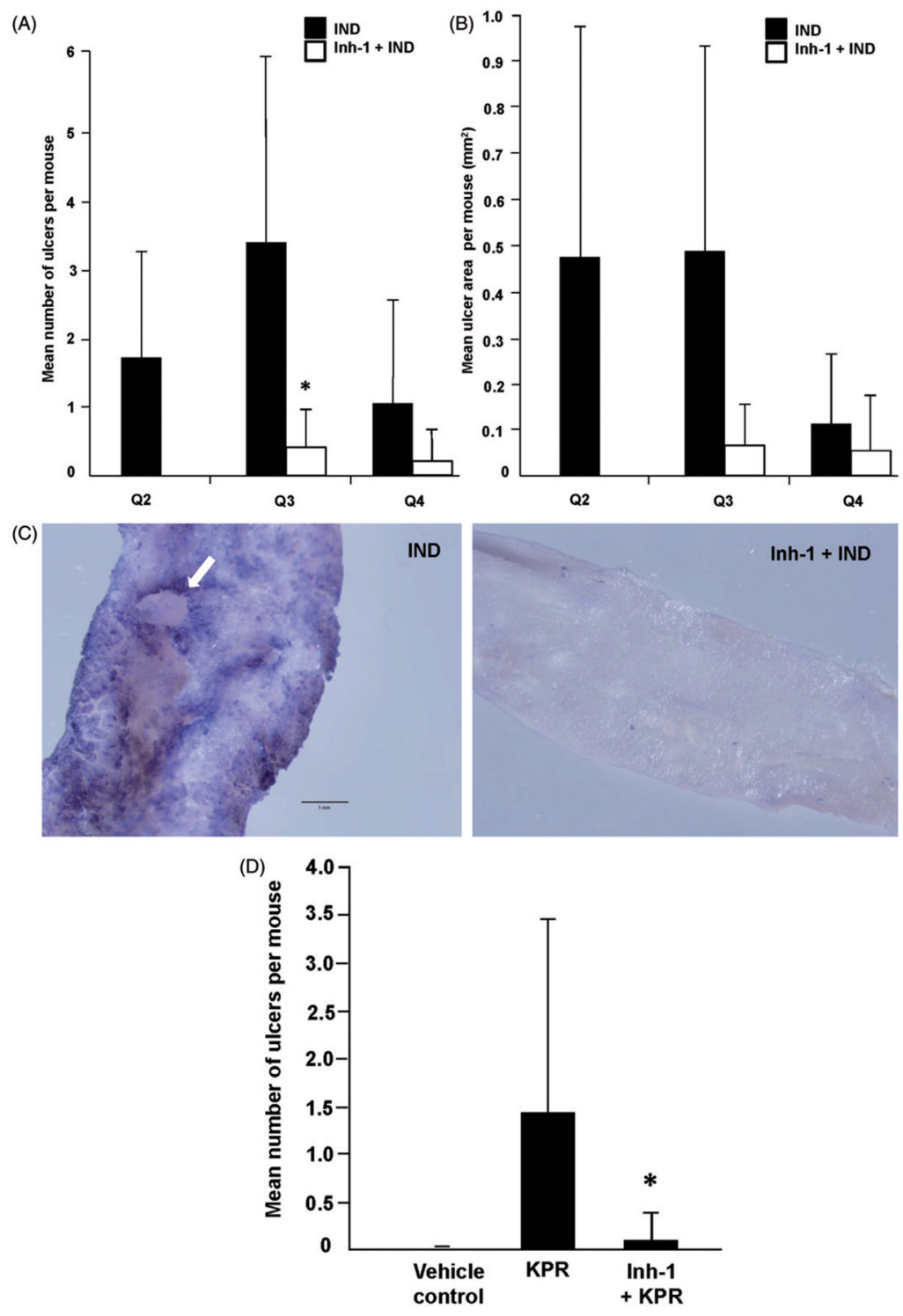

Figure 1.

Protective effects of Inhibitor-1 (Inh-1) on indomethacin (IND)-induced ulceration. Mice received a single dose of IND (10 mg/kg, ip) with or without pretreatment with Inh-1 (10 $\mu \mathrm{g} /$ mouse, bid, $\times 2$ days, last dose was given $1 \mathrm{~h}$ prior to IND). Ulcer numbers (A) and area (B) were determined in the four quartiles $(\mathrm{Q} 1-\mathrm{Q} 4)$ of the small intestine $18 \mathrm{~h}$ post-dose. Q1 did not exhibit any ulceration. Data are mean $\pm \mathrm{SD}(n=5$ mice per group. *, $p<0.05$ versus IND alone. (C) Micrograph of jejunal mucosa (luminal side) $18 \mathrm{~h}$ after IND treatment with or without Inh-1. Arrow, typical ulcer. The dark color of the nitroblue tetrazolium (NBT)stained intestinal tissue in the IND-treated mice is due to oxidoreductive stress converting the soluble NBT to the insoluble formazan. (D) Protective effect of Inh-1 on ketoprofen (KPR)-induced enteropathy. Mice received a single oral dose of KPR (100 mg/kg, ip) or vehicle, with or without pretreatment with Inh-1 $(10 \mu \mathrm{g} /$ mouse, bid, $\times 2$ days, last dose was given $1 \mathrm{~h}$ prior to KPR). Total ulcer numbers were determined $18 \mathrm{~h}$ post-dose. Data are mean $\pm \mathrm{SD}(n=10-11$ mice per group). *, $p<0.05$ versus KPR alone. 

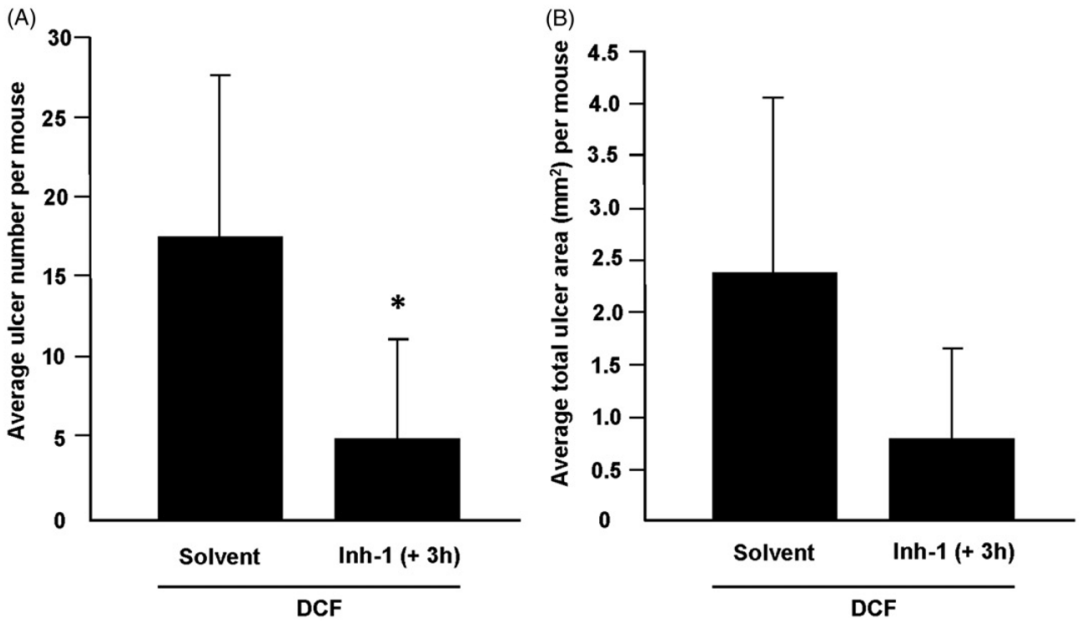

Figure 2.

Effects of post-treatment with Inh-1 on diclofenac (DCF)-induced small intestinal injury. Mice were given a single oral dose of Inh-1 (10 $\mu \mathrm{g} /$ mouse) or vehicle $(0.5 \%$ methyl cellulose) $3 \mathrm{~h}$ after a single intraperitoneal dose of DCF $(60 \mathrm{mg} / \mathrm{kg})$. The mice were euthanized $18 \mathrm{~h}$ after DCF, and the small intestinal mucosa processed for quantitative analysis of ulcer numbers (A) and total ulcer area per mouse, as determined by morphometry (B) across quartiles $1-4$. Data are mean $\pm \mathrm{SD}$ ( $n=5-6$ mice per group). *, $p<0.05$ versus DCF alone. 


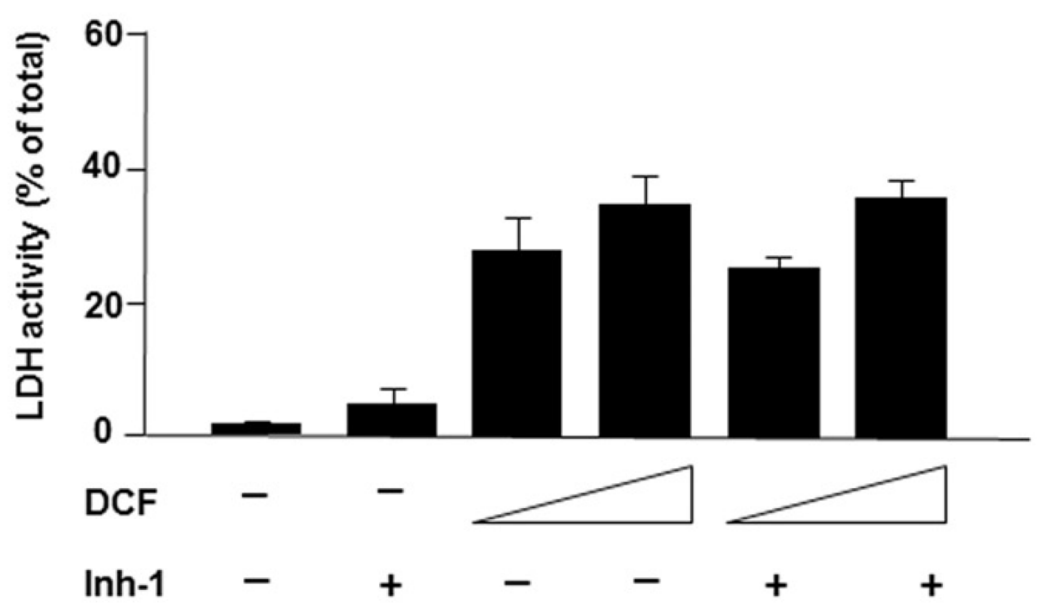

Figure 3.

Effects of Inh-1 on diclofenac (DCF)-induced acute cell injury in cultured mouse hepatocytes. Overnight-precultured primary mouse hepatocytes were incubated with DCF $(500$ or $1000 \mu \mathrm{M})$ in the presence or absence of Inh-1 $(100 \mu \mathrm{M})$, and LDH activity in the culture media was assessed after $24 \mathrm{~h}$ exposure $(100 \%=$ both intra- and extracellular LDH activity). Data are mean \pm SD of triplicate wells using two independent cell preparations. 

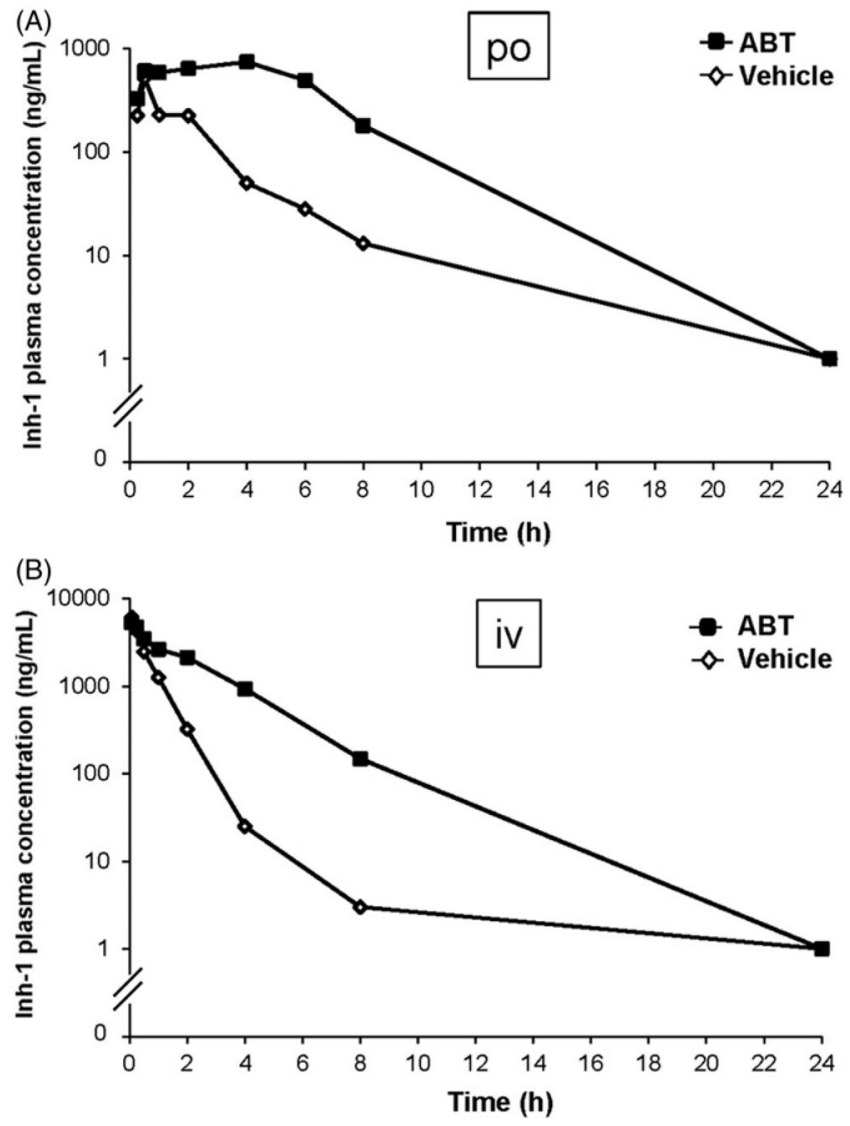

Figure 4.

Mean plasma concentration-time profiles of Inh-1 following a single oral dose (A) or a single intravenous dose (B). Inhibitor- 1 was administered at a dose of $3 \mathrm{mg} / \mathrm{kg}$ to mice with or without pretreatment with 1-aminobenzotriazole (ABT, $50 \mathrm{mg} / \mathrm{kg}$, po) which was given 2 $\mathrm{h}$ prior to Inh-1. Filled symbols, ABT; white symbols, vehicle. Data points are mean of three mice. 


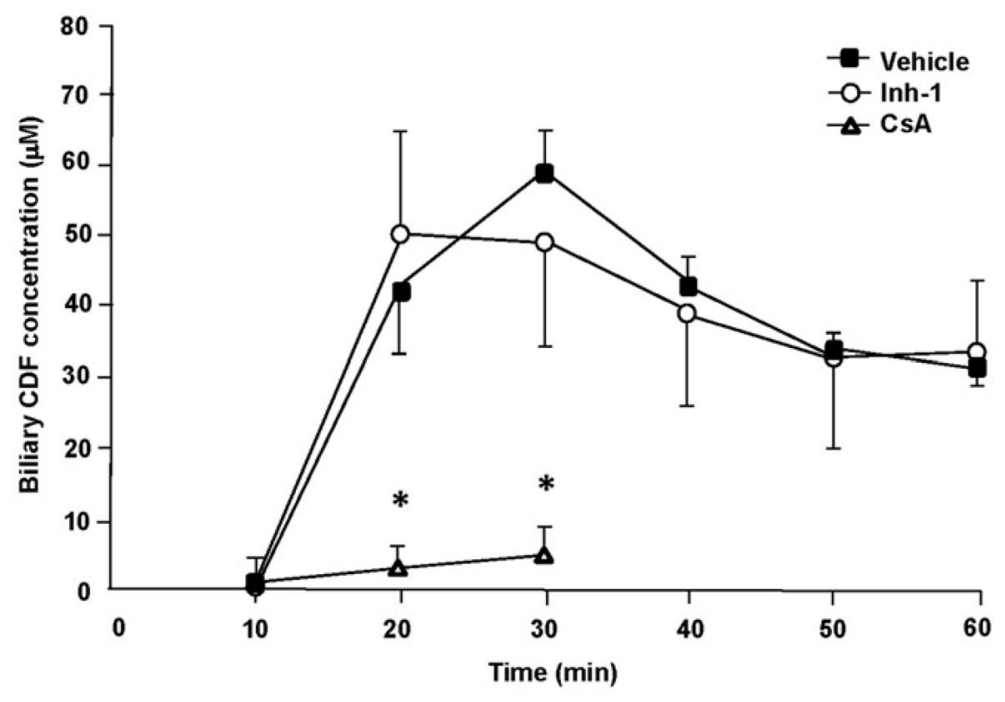

Figure 5.

Effect of Inhibitor-1 on the hepatobiliary excretion rate of the Mrp2 substrate, 5 (and 6)carboxy-2 $2^{\prime}, 7^{\prime}$-dichlorofluorescein (CDF). Mice were pretreated with Inh-1 or vehicle as described in the section "Materials and methods". Following anesthesia, the gall bladder was cannulated, and bile flow was monitored until it was stable. The mice received a single injection of CDF diacetate $(1.5 \mu \mathrm{mol} / \mathrm{mouse})$, and bile was collected at 10 -min intervals. CDF concentrations were determined by fluorescence spectrophotometry; bile flow was determined gravimetrically (assuming a specific mass of bile of 1.0). Data are mean \pm SD ( $n$ $=5$ mice per group). ${ }^{*}, p<0.05$ versus vehicle control. 


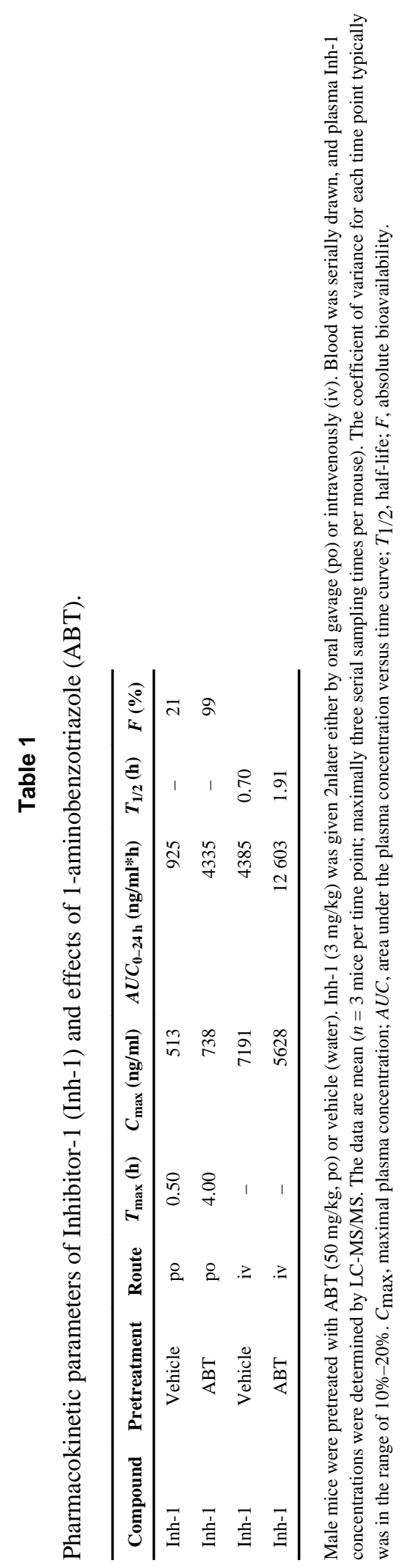

Xenobiotica. Author manuscript; available in PMC 2014 April 02. 